\title{
Andreev-reflection spectroscopy of ferromagnets: the impact of Fermi surface mismatch
}

\author{
Elina Tuuli ${ }^{1,2,3}$ and Kurt Gloos ${ }^{1,3}$ \\ ${ }^{1}$ Wihuri Physical Laboratory, Department of Physics and Astronomy, \\ University of Turku, FIN-20014 Turku, Finland \\ ${ }^{2}$ Graduate School for Materials Research, \\ (GSMR), FIN-20500 Turku, Finland and \\ ${ }^{3}$ Turku University Centre for Materials and Surfaces (MatSurf), FIN-20014 Turku, Finland*
}

(Dated: December 5, 2018)

\begin{abstract}
We have investigated point contacts between a superconductor $\left(\mathrm{Nb}, \mathrm{AuIn} \mathrm{In}_{2}\right)$ and a normal metal (ferromagnetic $\mathrm{Co}$, non-magnetic $\mathrm{Cu}$ ). The observed Andreev-reflection spectra were analyzed using the modified BTK theory including spin-polarization effects. This resulted in a polarization of Co that agrees with observations by others, but lifetime effects describe the spectra equally well. On the other hand, the spectra with non-magnetic $\mathrm{Cu}$ can be well described using the spin-polarization model. The ambiguity between polarization and lifetime interpretation poses a dilemma which can be resolved by considering the normal reflection at those interfaces due to Fermi surface mismatch. Our data suggest that Andreev reflection at $\mathrm{Nb}$ - Co contacts does deliver the true magnetic polarization of Co only when lifetime effects and the above mentioned intrinsic normal reflection are included.
\end{abstract}

PACS numbers: 74.45.+c, 72.25.Mk, 73.40.-c, 85.30.Hi, 75.50.Cc

*Electronic address: estuul@utu.fi 


\section{INTRODUCTION}

Andreev-reflection spectroscopy at point contacts has been suggested as a versatile tool to determine the magnetic (spin current) polarization $P$ of ferromagnets [1, 2]. Today it is widely believed [3-11] that the polarization can be reliably extracted from the measured point-contact spectra by applying a modified version of the BTK theory of Andreev reflection [12], like Strijkers' [13] or Mazin's model [14]. However, it has also been noted that the interpretation of these point-contact spectra presents extra difficulties because of spurious superposed anomalies and the poor convergence of the fitting procedure [7, 9, 11].

Andreev reflection across a ballistic contact between a normal metal and a superconductor requires the transfer of an electron pair with opposite momentum and spin from the normal conductor to form a Cooper pair in the superconductor. In an equivalent description an electron is transferred to the superconductor and the corresponding hole is retro-reflected. This reduces the contact resistance by a factor of two for energies within the superconducting gap $2 \Delta$. Normal reflection at the interface has a pronounced effect on the shape of the spectra because it enters the pair transfer twice by affecting the incident electron and also the retroreflected hole, yielding the typical double-minimum structure of Andreev reflection. To keep the number of adjustable parameters as small as possible, Blonder, Tinkham, and Klapwijk [12] described normal reflection by a $\delta$-function barrier of strength $Z$. The BTK theory is well accepted to analyze the Andreev-reflection spectra of ballistic contacts between BCS-type superconductors and non-magnetic normal metals.

Since interfaces are usually not perfect, they cause additional scattering that can break up the Cooper pairs and, thus, reduce the superconducting order parameter. Dynes' model describes this situation by a finite lifetime $\tau=\hbar / \Gamma$ of the Cooper pairs, which strongly reduces the magnitude of the Andreev-reflection anomaly [15].

A magnetically polarized metal has an unequal number of spin up and spin down electrons. Conduction electrons that can not find their corresponding pair with opposite spin do not participate in Andreev reflection, opening the way to directly measure the polarization [1, 2]. The polarization reduces the magnitude of the Andreev-reflection anomaly like the lifetime effects, and it leads to a zero-bias maximum of the differential resistance similar to that of normal reflection. With few exceptions [8, 9], the analysis of superconductor - ferromagnet spectra usually excludes lifetime effects [3-7, 10, 11] so that $P$ and $Z$ are the only main 
adjustable parameters. Also the superconducting energy gap has to be treated as a variable, although its approximate value at the contact is known from the bulk superconducting properties. Often a so-called 'broadening parameter' is included to improve the fit quality by simulating an enhanced smearing of the Fermi edge [5, 7, 11], but increasing the parameter number means the solution becomes more easily degenerate.

We show here that the Andreev-reflection spectra of both ferromagnets and non-magnets can be fitted equally well by assuming a magnetic polarization of the normal metal without lifetime effects and vice versa. This problem can be solved by taking into account the lower bound of $Z$ due to Fermi surface mismatch.

\section{EXPERIMENTAL}

Our experiments are based on shear contacts between superconducting $\mathrm{Nb}\left(T_{c}=9.2 \mathrm{~K}\right)$ and normal conducting $\mathrm{Co}$ and $\mathrm{Cu}$ wires $(\sim 0.25 \mathrm{~mm}$ diameter $)$ at $4.2 \mathrm{~K}$ in liquid helium. Co is a band-ferromagnet with $T_{\text {Curie }}=1388 \mathrm{~K}$ and $\mathrm{Cu}$ a non-magnetic normal metal [16]. We have also re-analyzed older spear-anvil type experiments at $0.05 \mathrm{~K}$ with the BCS-type superconductor $\mathrm{AuIn}_{2}\left(T_{c}=0.21 \mathrm{~K}\right)$ in contact with a $\mathrm{Cu}$ wire [17]. The differential resistance $d V / d I$ was measured as function of bias voltage $V$ with low-frequency current modulation in four-wire mode.

Figure 1 shows typical spectra of $\mathrm{Nb}$ - Co as well as $\mathrm{Nb}$ - Cu contacts. We have observed various types that can be classified as follows: $i$ ) Andreev-reflection double minimum (a, d), ii) Andreev reflection with side peaks (b, e), iii) single zero-bias minimum with or without side peaks (c, f), and $i v$ ) zero-bias maximum without signs of superconductivity (not shown). For our analysis we have used only contacts of type $i$ ) and $i i$ ) which show the 'hallmark' of Andreev reflection. The origin of the side peaks will be discussed elsewhere. Contacts of type $i v$ ) were studied earlier [18].

\section{DISCUSSION}

We fitted the spectra in the conventional way [3 11] using Strijkers' model and assuming $\Gamma=0$ (Figure 2). Mazin's model would only slightly change the $P(Z)$ data [19]. The resultant polarization of $\mathrm{Nb}$ - Co contacts in Figure 3 (a) agrees well with that found 


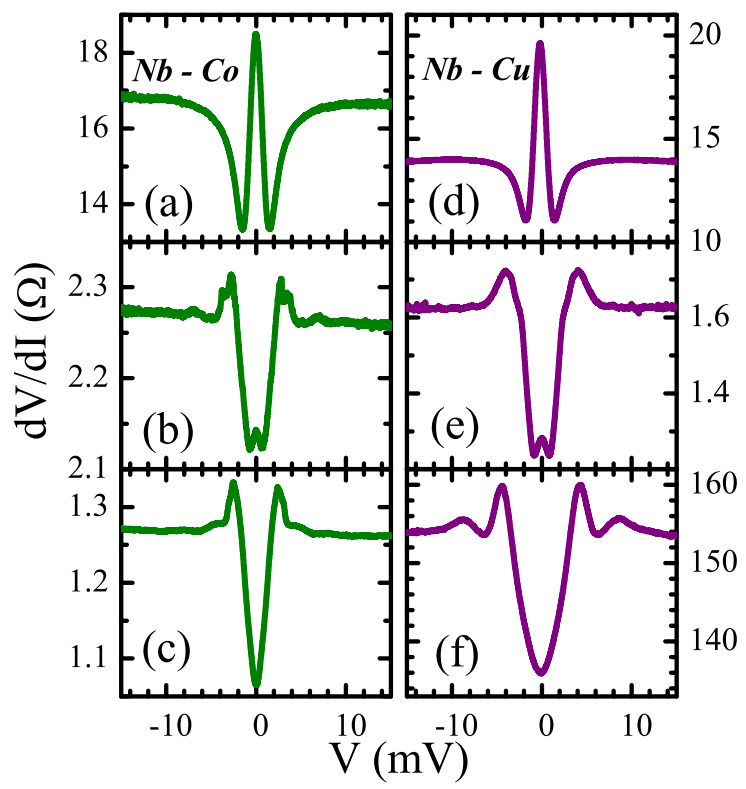

FIG. 1: (Color online) Typical $d V / d I(V)$ spectra of (a - c) Nb - Co and (d - f) Nb - Cu contacts at $4.2 \mathrm{~K}$.

by others [5, 11, 13]. However, analysing the $\mathrm{Nb}-\mathrm{Cu}$ and the $\mathrm{AuIn}_{2}-\mathrm{Cu}$ spectra in the same way, assuming $\Gamma=0$ and allowing $P$ to vary, yielded almost the same $P(Z)$ as for the $\mathrm{Nb}$ - Co contacts (Figure 3): Without advance knowledge that $\mathrm{Cu}$ has zero spin polarization $P=0$, we would be led to believe that it is actually polarized like ferromagnetic Co. Such a possibility was mentioned - but discarded - by Chalsani et al. [9] for $\mathrm{Pb}$ $\mathrm{Cu}$ contacts. Nevertheless, this speculation could be supported by recent experiments on the size-dependence of the so-called zero-bias anomaly which has been attributed to the spontaneous electron spin polarization at the point contact [18].

It appears trivial to assume $P=0$ for $\mathrm{Cu}$ and to use the lifetime parameter $\Gamma$ that fits the observed spectra equally well. But the lifetime-only model also works well for ferromagnetic Co, as demonstrated in Figure 2 where the theoretical curves for the two fitting procedures can be barely separated.

In order to study the similarities and differences between the two models in more detail, we have calculated spectra at small, medium, and large values of $Z$ together with their typical polarization as found in the experiments summarized in Figure 3. These theoretical curves were then fitted with the lifetime-only model. Figure 4 demonstrates the perfect agreement between the two models at large $Z$ and small $P$. This confirms earlier findings by 

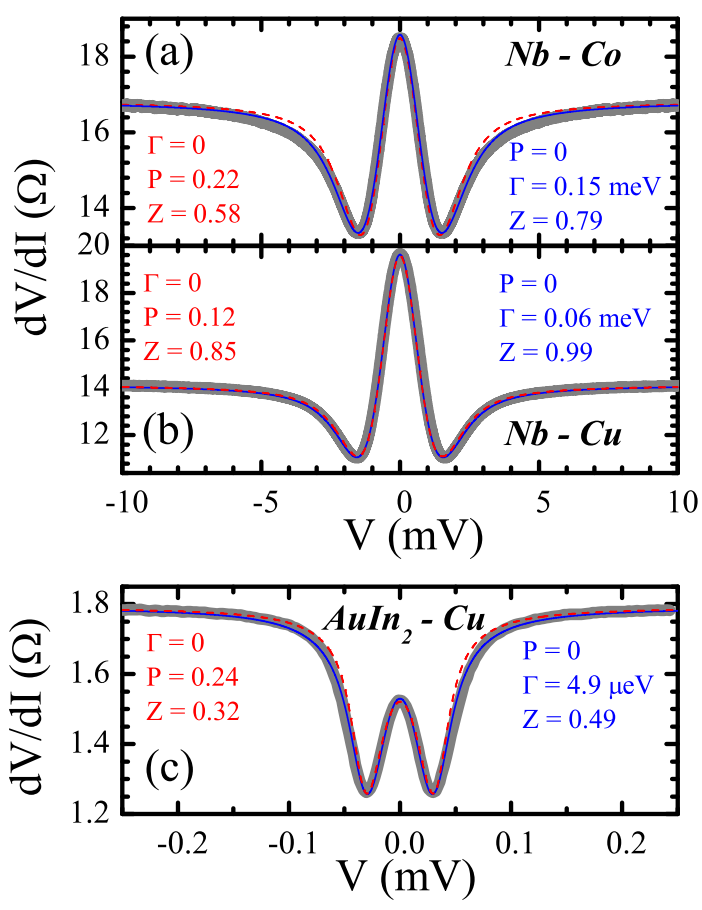

FIG. 2: (Color online) Typical differential resistance versus bias voltage (thick solid lines) together with fits derived by assuming $\Gamma=0$ (thin dashed lines) and $P=0$ (thin solid lines) using the indicated fitting parameters. For all contacts the curves are almost indistinguishable. Deviations found only near the shoulder where $d V / d I$ starts to drop from its normal-state value can be removed by introducing a 'broadening parameter'. (a) $\mathrm{Nb}-$ Co at $T=4.2 \mathrm{~K}$ and $2 \Delta=2.6 \mathrm{meV}$, (b) $\mathrm{Nb}-$ $\mathrm{Cu}$ at $T=4.2 \mathrm{~K}$ and $2 \Delta=2.5 \mathrm{meV}$, and (c) $\mathrm{AuIn}_{2}-\mathrm{Cu}$ at $T=0.05 \mathrm{~K}$ and $2 \Delta=65 \mu \mathrm{eV}$.

Chalsani et al. in the case of $\mathrm{Pb}-\mathrm{Cu}$ and $\mathrm{Pb}$ - Co contacts [9]. Deviations become obvious only at small $Z$ and large $P$. Note also that the strong $Z$-dependence of $P$ turns into a $\Gamma$ at nearly constant $Z$, in agreement with the experimental data in Figure 3. Consequently, distinguishing lifetime effects from the magnetic polarization requires additional information.

This knowledge could be obtained from normal reflection: Figure 3 shows that the $P(Z)$ data are almost evenly distributed on the $Z$ axis from $Z \approx 0$ to the maximum value of $Z \approx 0.8$ for $\mathrm{Nb}-\mathrm{Co}$ and $\mathrm{Nb}-\mathrm{Cu}$ contacts. In contrast, the $\Gamma(Z)$ data are centered at around $Z \approx 0.8$, indicating a preferred value for normal reflection. This different behaviour must have a reason.

$Z$ consists of two parts, $Z_{\text {barrier }}$ describes reflection at a possible interface tunneling barrier (and any other mechanism that might be subsumed under this term), and $Z_{0}$ due a mismatch of the Fermi surfaces or band structures of the two electrodes. In free-electron approximation 


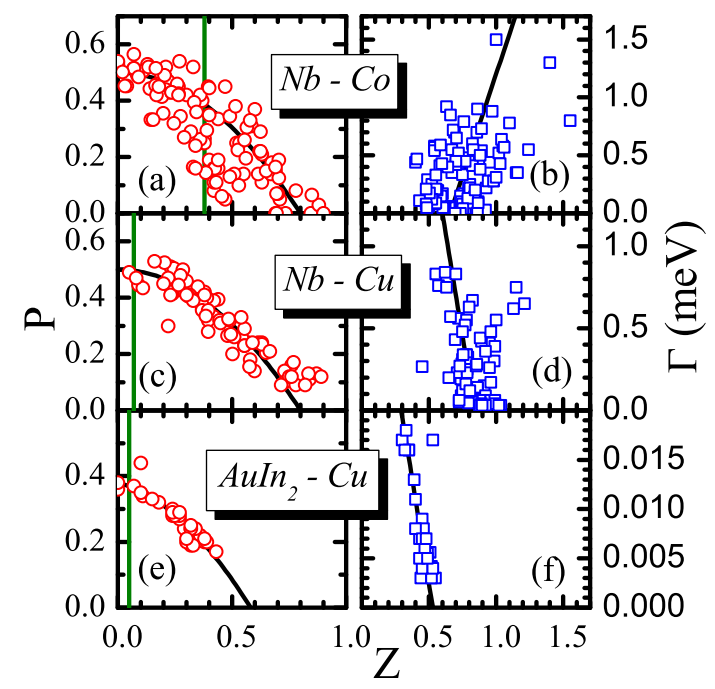

FIG. 3: (Color online) Polarization $P$ at $\Gamma=0$ and life-time broadening $\Gamma$ at $P=0$ versus $Z$ of $\mathrm{Nb}$ - $\mathrm{Co}, \mathrm{Nb}-\mathrm{Cu}$, and $\mathrm{AuIn}_{2}-\mathrm{Cu}$ contacts. The vertical solid lines represent the expected minimum $Z_{0}$ due to Fermi momentum mismatch in free-electron approximation. Solid lines through the data points serve as guide to the eye.

Fermi surface mismatch reduces to a mismatch $r=v_{F 1} / v_{F 2}$ of Fermi velocities $v_{F 1,2}$ on both sides of the contact and results in [20]

$$
Z^{2}=Z_{\text {barrier }}^{2}+Z_{0}^{2}=Z_{\text {barrier }}^{2}+\frac{(1-r)^{2}}{4 r}
$$

Thus $Z_{0}$ defines a lower bound of $Z$ when a tunneling barrier is absent. That means, without tunneling barrier the $Z$ parameter of the contacts for a given metal combination should be constant while a tunneling barrier would add a tail to the $Z$ distribution at large values. The experimental data in Figure 3 indicate that our contacts either have a negligibly small $Z_{0}$ plus an irreproducible tunneling barrier (polarization-only model) or a large $Z_{0}$ with a negligibly small tunneling barrier (lifetime-only model).

Note that Eq. 1 requires equal effective electron masses. For example, Fermi velocity mismatch is negligible at interfaces between a heavy-fermion compound and a simple metal because their huge velocity mismatch of up to $r \approx 1000$ is compensated by the large mismatch of the effective electron masses [21]. Therefore it is more appropriate to speak of a momentum mismatch instead and replace the variable $r$ by the ratio of Fermi wave numbers $k_{F 1,2}$.

While we do not know whether our point contacts possess a tunneling barrier, it should 


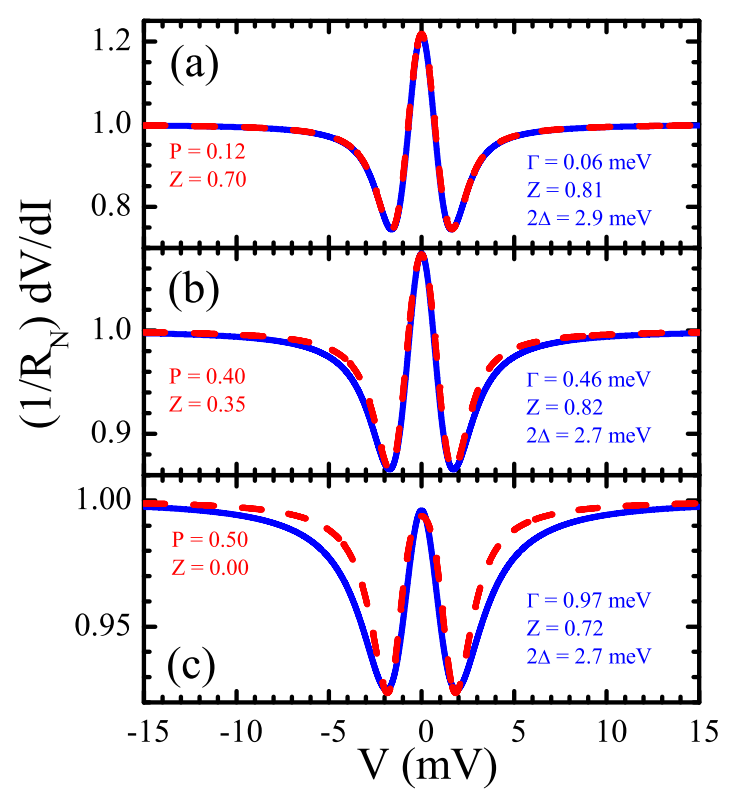

FIG. 4: (Color online) Comparison between the polarization-only (red dashed lines) and lifetimeonly (blue solid lines) models for contacts with (a) small, (b) medium, and (c) large polarization. The differential resistance $d V / d I$ is normalized to the normal contact resistance $R_{N}$. First the polarization-only spectra were calculated assuming the indicated $P$ and $Z$ at $2 \Delta=3.0 \mathrm{meV}$ for niobium and $T=4.2 \mathrm{~K}$. Then the lifetime-only spectra were fitted, resulting in the indicated $\Gamma$ and $Z$. For this fitting the energy gap had to be slightly adjusted.

be possible to predict $Z_{0}$ from the known band structure of metals. This turns out to be quite difficult because there are different theoretical and experimental estimates for the Fermi surface properties. In free-electron approximation $k_{F}=13.6 \mathrm{~nm}^{-1}$ for $\mathrm{Cu}$ and $k_{F}=$ $11.8 \mathrm{~nm}^{-1}$ for $\mathrm{Nb}[16]$. AuIn 2 has nearly the same conduction electron density as $\mathrm{Cu}$ and, thus, a very similar $k_{F}$ [22]. Co has spin-split energy bands, and therefore different wave numbers for the two spin directions. Its average Fermi velocity $v_{F} \approx 280 \mathrm{~km} / \mathrm{s}$ is known from critical-current oscillations in Josephson $\pi$-junctions [24]. Its effective electron mass $m$ is about twice the free electron mass [25], yielding $k_{F}=m v_{F} / \hbar \approx 5.6 \mathrm{~nm}^{-1}$. The minimum $Z$ parameters $Z_{0} \approx 0.05$ for $\mathrm{AuIn}_{2}-\mathrm{Cu}$ [17], $Z_{0} \approx 0.07$ for $\mathrm{Nb}-\mathrm{Cu}$, and $Z_{0} \approx 0.38$ for $\mathrm{Nb}$ - Co are consistent with the polarization-only and with the lifetime-only model for $\mathrm{Nb}-\mathrm{Cu}$ and $\mathrm{AuIn}_{2} \mathrm{Cu}$, but they clearly contradict the conventional polarization data of $\mathrm{Nb}$ - Co. On the other hand, $\mathrm{Nb}$ is claimed [23] to have a Fermi velocity of only $v_{F}=273 \mathrm{~km} / \mathrm{s}$, based on critical field measurements, with a heat-capacity derived effective mass enhancement of 
about 2. That would mean a perfect match between $\mathrm{Nb}$ and Co with $Z_{0} \approx 0$.

Quite different estimates for $Z_{0}$ come from proximity-effect studies on $\mathrm{Nb}$ - normal metal bi-layers [26-28] with interface transparencies $1 /\left(1+Z^{2}\right)$ consistently smaller than $50 \%$. Since those bi-layers should have no (oxide) tunneling barrier, their Fermi surface mismatch must be large with $Z_{0} \geq 1$ for non-magnetic normal metals $\mathrm{Cu}, \mathrm{Ag}, \mathrm{Al}$, and $\mathrm{Pd}$ as well as for the ferromagnets $\mathrm{Fe}$ and $\mathrm{Ni}$. The same is to be expected for Nb - Co interfaces [29]. This is difficult to reconcile with the standard interpretation of Andreev-reflection spectroscopy of the ferromagnets - here lifetime effects would fit much better.

If we assume that our $\mathrm{Nb}-\mathrm{Cu}$ contacts are non-magnetic, then they deliver the normal reflection $Z_{0} \approx 0.8$ due to Fermi surface mismatch in good agreement with the above mentioned proximity-effect data where a tunneling barrier can be excluded. The scattering $\Delta Z \approx \pm 0.2$ around the average could result, for example, from small residual oxide barriers or the different crystallographic orientations of the polycrystalline electrodes when the contacts are formed. There is little reason to assume that Nb-Co contacts should have a much smaller Fermi surface mismatch even down to $Z_{0} \approx 0$. The $P(Z)$ data points of Nb-Co at small $Z$ are therefore invalid. Shifting them to higher $Z$ values requires the inclusion of lifetime effects, a quite natural consequence since we would expect the interface with ferromagnetic Co not to be less pair breaking than the one with non-magnetic $\mathrm{Cu}$. However, without precise knowledge of $Z$ it is difficult to extract any reliable value of the polarization. Our data even show that the Nb-Co contacts could be non-magnetic like the Nb-Cu contacts. A small polarization at contacts with a large $Z$ would be consistent with predictions of the conventional theory [5].

On the other hand, we can not exclude that $\mathrm{Nb}$ - $\mathrm{Cu}$ contacts are magnetic. The Andreevreflection spectra are consistent with a small local polarization of $\mathrm{Cu}$ as has been suggested in Ref. [18].

We have obtained similar Andreev-reflection data for the ferromagnets Fe and Ni as well as the non-magnets $\mathrm{Ag}$ and $\mathrm{Pt}$ in contact with $\mathrm{Nb}$, indicating a rather general problem of Andreev-reflection spectroscopy. 


\section{CONCLUSIONS}

The available information suggests that the true (spin current) polarization of the ferromagnets is probably not that derived from Andreev-reflection spectra when lifetime effects are arbitrarily excluded and the intrinsic normal reflection due to Fermi surface mismatch ignored.

\section{Acknowledgments}

We thank the Jenny and Antti Wihuri Foundation for financial support.

[1] M. J. M. de Jong and C. W. J. Beenakker, Phys. Rev. Lett. 74, 1657 (1995).

[2] R. J. Soulen Jr., J. M. Byers, M. S. Osofsky, B. Nadgorny, T. Ambrose, S. F. Cheng, P. R. Broussard, C. T. Tanaka, J. Nowack, J. S. Moodera, A. Barry, and J. M. D. Coey, Science 282, 85 (1998).

[3] S. K. Upadhyay, A. Palanisami, R. N. Louie, and R. A. Buhrman, Phys. Rev. Lett. 81, 3247 (1998).

[4] Y. Ji, G. J. Strijkers, F. Y. Yang, C. L. Chien, J. M. Byers, A. Anguelouch, Gang Xiao, and A. Gupta, Phys. Rev. Lett. 86, 5585 (2001).

[5] C. H. Kant, O. Kurnosikov, A. T. Filip, P. LeClair, H. J. M. Swagten, and W. J. M. de Jonge, Phys. Rev. B 66, 212403 (2002).

[6] N. Auth, G. Jakob, T. Block, and C. Felser, Phys. Rev. B 68, 024403 (2003).

[7] Y. Bugoslavsky, Y. Miyoshi, S. K. Clowes, W. R. Branford, M. Lake, I. Brown, A. D. Caplin, and L. F. Cohen, Phys. Rev. B 71, 104523 (2005).

[8] S. Mukhopadhyay, P. Raychaudhuri, D. A. Joshi, and C. V. Tomy, Phys. Rev. B 75, 014504 (2007).

[9] P. Chalsani, S. K. Upadhyay, O. Ozatay, and R. A. Buhrman, Phys. Rev. B 75, 094417 (2007).

[10] M. Stokmaier, G. Goll, D. Weissenberger, C. Sürgers, and H. v. Löhneysen, Phys. Rev. Lett. 101, 147005 (2008).

[11] V. Baltz, A. D. Naylor, K. M. Seemann, W. Elder, S. Sheen, K. Westerholt, H. Zabel, G. 
Burnell, C. H. Marrows, and B. J. Hickey, J. Phys.: Condens. Matter 21, 095701 (2009).

[12] G. E. Blonder, M. Tinkham, and T. M. Klapwijk, Phys. Rev. B 25, 4515 (1982).

[13] G. J. Strijkers, Y. Ji, F. Y. Yang, C. L. Chien, and J. M. Byers, Phys. Rev. B 63, 104510 (2001).

[14] I. I.Mazin, A. A. Golubov, and B. Nadgorny, J. Appl. Phys. 89, 7576 (2001).

[15] A. Pleceník, M. Grajcar, S̆. Ben̆ac̆ka, P. Seidel, and A. Pfuch, Phys. Rev. B 49, 10016 (1994).

[16] N. W. Ashcroft and N. D. Mermin Solid State Physics (Thomson Learning, 1976).

[17] K. Gloos and F. Martin, Z. Phys. B - Condensed Matter 99, 321 (1996).

[18] K. Gloos, Low Temp. Phys. 35, 935 (2009).

[19] Y. Ji, G. J. Strijkers, F. Y. Yang, and C. L. Chien, Phys. Rev. B 64, 224425 (2001).

[20] G. E. Blonder and M. Tinkham, Phys. Rev. B 27, 112 (1983).

[21] G. Deutscher and P. Nozières, Phys. Rev. B 50, 13557 (1994).

[22] J. A. Rayne, Phys. Lett. 7, 114 (1963).

[23] H. R. Kerchner, D. K. Christen, and S. T. Sekula, Phys. Rev. B 24, 1200 (1981).

[24] J. W. A. Robinson, S. Piano, G. Burnell, C. Bell, and M. G. Blamire, Phys. Rev. Lett. 97, $177003(2006)$.

[25] G. J. McMullan, D. D. Pilgram, and A. Marshall, Phys. Rev. B 46, 3789 (1992).

[26] C. Cirillo, S. L. Prischepa, M. Salvato, and C. Attanasio, Eur. Phys. J. B 38, 59 (2004).

[27] A. Tesauro, A. Aurigemma, C.Cirillo, S. L. Prischepa, M. Salvato, and C. Attanasio, Supercond. Sci. Technol. 18, 1 (2005).

[28] C. Attanasio in: R. Gross, A. Sidorenko, and L. Tagirov (Eds.) Nanoscale Devices - Fundamentals and Applications (Springer, 2006), pp 241.

[29] J. Liang, S. F. Lee, W. T. Shih, W. L. Chang, C. Yu, and Y. D. Yao, J. Appl. Phys. 92, 2624 (2002). 